\title{
Thermodynamic framework for a generalized heat transport equation
}

\author{
Yangyu Guo, Moran Wang*
}

Key Laboratory for Thermal Science and

Power Engineering of Ministry of Education,

Department of Engineering Mechanics, Tsinghua University, Beijing, China

*Email address for correspondence: mrwang@tsinghua.edu.cn

Communicated by Vito Antonio Cimmelli and David Jou

Received on December 25, 2014. Accepted on May 4, 2015.

\begin{abstract}
In this paper, a generalized heat transport equation including relaxational, nonlocal and nonlinear effects is provided, which contains diverse previous phenomenological models as particular cases. The aim of the present work is to establish an extended irreversible thermodynamic framework, with generalized expressions of entropy and entropy flux. Nonlinear thermodynamic force-flux relation is proposed as an extension of the usual linear one, giving rise to the nonlinear terms in the heat transport equation and ensuring compatibility with the second law. Several previous results are recovered in the linear case, and some additional results related to nonlinear terms are also obtained.

Keywords: generalized heat transport equation; extended irreversible thermodynamics; generalized entropy flux; nanoscale heat transport.

AMS subject classification: 05A16, 65N38, 78M50.
\end{abstract}

\section{Introduction}

Heat transport has been a phenomenon of basic interest for the foundation of thermodynamics since the beginning of this physical theory. In the early years of thermodynamics, two different views about the nature of heat competed with each other [1]: heat as a conserved substance (dubbed as caloric) and heat as a form of energy. It was the latter view which enforced the first mathematical statement of the second law by Clausius and Thomson [1]. Later, classical linear irreversible thermodynamics [2] was developed by Onsager [3,4], Eckart [5,6], Meixner [7] and Prigogine [8] for the near-equilibrium heat transport described by Fourier law. In recent years, generalized laws of heat transport in micro- and nanoscale systems [9-14] have been again the stimulus for further developments of compatible irreversible thermodynamics and wider formulations of the second law, as in 
diverse branches of rational thermodynamics [15], rational extended thermodynamics [16-19], extended irreversible thermodynamics [20,21], weakly nonlocal thermodynamics [22,23] and GENERIC [24-26]. In the present work, we will illustrate the close connection between generalized heat transport equations and generalized forms of the second law in the framework of extended irreversible thermodynamics. In a previous paper [27], we have proposed a generalized heat transport equation including relaxational, nonlocal and nonlinear terms:

$$
\begin{aligned}
\tau \frac{\partial \mathbf{q}}{\partial t}+\mathbf{q}= & -\lambda \nabla T+m_{1} \mathbf{q} \nabla \cdot \mathbf{q}+m_{2} \mathbf{q} \cdot \nabla \mathbf{q}+m_{3} \nabla \mathbf{q}^{2}+m_{4} \nabla^{2} \mathbf{q} \\
& +m_{5} \nabla(\nabla \cdot \mathbf{q})+m_{6} \mathbf{q}(\mathbf{q} \cdot \nabla T)+m_{7} \mathbf{q}^{2} \nabla T
\end{aligned}
$$

and identified the coefficients $m_{i}(i=1, \ldots, 7)$ by comparing Eq. (1) with previous macroscopic heat transport equations achieved in diverse different phenomenological approaches, such as phonon hydrodynamics model $[21,28,29]$ with $m_{1}=m_{2}=m_{3}=m_{6}=m_{7}=0$ and $m_{4}, m_{5} \neq 0$, dualphase-lagging model [11,30] with $m_{1}=m_{2}=m_{3}=m_{4}=m_{6}=m_{7}=0$ and $m_{5} \neq 0$, the thermon gas model [31,32] with $m_{3}=m_{4}=m_{5}=m_{7}=0$ and $m_{1}, m_{2}, m_{6} \neq 0$. Furthermore, we have related such coefficients to the phase speed of high-frequency heat waves along nonequilibrium steady states in several different situations. Each coefficient has a particular physical meaning and particular physical consequences, in such a way that Eq. (1) is not merely a formal equation, but it provides a synthesis of a number of different theories motivated by different physical phenomena.

Here, we try to establish a thermodynamic framework for Eq. (1), namely, we seek for the expressions of entropy and entropy flux which make Eq. (1) compatible with the second law, i.e. ensuring a positive-definite entropy production. The answer to the problem is not obvious, because neither the expression of entropy nor entropy flux are known a priori, but are closely related to the heat transport equation. We will follow the general lines in the framework of extended irreversible thermodynamics (EIT), but with some nonlinear extensions beyond the usual linear thermodynamic force-flux relations.

\section{Entropy, entropy flux and second law}

In this section and following, the internal energy $u$, heat flux $\mathbf{q}$, and flux of heat flux $\mathbf{Q}$ are taken as basic state variables [21]. In contrast to $u$ and $\mathbf{q}, \mathbf{Q}$ has not an established and clear physical meaning, but it has a microscopic physical basis in kinetic theory and a rigorous formal meaning, thus having been used in many occasions in the literature $[19,21,33,34]$. 


\section{Generalized irreversible thermodynamics}

For the sake of generality, we will discuss the expression of $\mathbf{Q}$ in terms of nonlocal terms as $\nabla \mathbf{q}$ and nonlinear terms as $\mathbf{q q}$ in Section 3, but for the moment we leave $\mathbf{Q}$ as open as possible. Thus the generalized specific entropy is expressed as $s=s(u, \mathbf{q}, \mathbf{Q})$ and the corresponding generalized Gibbs relation becomes [21]:

$$
d s=\theta^{-1} d u-v \alpha_{1} \mathbf{q} \cdot d \mathbf{q}-v \alpha_{2} \mathbf{Q}: d \mathbf{Q},
$$

with $v$ the specific volume, $\alpha_{1}$ and $\alpha_{2}$ coefficients whose physical meaning will be explored below, and $\theta$ the nonequilibrium absolute temperature.

Combining Eq. (2) with the energy balance equation:

$$
\rho \dot{u}=-\nabla \cdot \mathbf{q}
$$

we obtain:

$$
\rho \dot{s}=-\theta^{-1} \nabla \cdot \mathbf{q}-\alpha_{1} \mathbf{q} \cdot \dot{\mathbf{q}}-\alpha_{2} \mathbf{Q}: \dot{\mathbf{Q}} .
$$

The entropy balance equation and the expression of entropy flux are respectively $[2,21]$ :

$$
\rho \dot{s}=-\nabla \cdot \mathbf{J}^{s}+\sigma^{s},
$$

$$
\mathbf{J}^{s}=\frac{\mathbf{q}}{\theta}+\beta \mathbf{Q} \cdot \mathbf{q},
$$

where $\beta$ is a coefficient which according to information theory, would be $\beta=-v \alpha_{1}$ [21]. Eq. (5) is a general expression of a balance law, whereas Eq. (6) is a particular hypothesis concerning the form of the entropy flux, which turns out to be far more general than the classical expression $\mathbf{J}^{s}=\mathbf{q} / T$. Analyses of generalized expressions of entropy flux may be found in $[21,22$, $35-42]$.

Substitution of Eq. (4) and Eq. (6) into Eq. (5) gives rise to the expression of entropy production:

$$
\sigma^{s}=\mathbf{q} \cdot X_{\mathbf{q}}+\mathbf{Q}: X_{\mathbf{Q}},
$$

with the conjugate thermodynamic forces to thermodynamic fluxes $\mathbf{q}$ and $\mathbf{Q}$ respectively given by:

$$
X_{\mathbf{q}}=-\alpha_{1} \dot{\mathbf{q}}+\nabla\left(\theta^{-1}\right)+\beta \nabla \cdot \mathbf{Q},
$$

$$
X_{\mathbf{Q}}=-\alpha_{2} \dot{\mathbf{Q}}+\beta \nabla \mathbf{q}
$$


Eq. (3) acting as the evolution equation for the state variable $u$, we must also obtain similar evolution equations for $\mathbf{q}$ and $\mathbf{Q}$ :

$$
\dot{\mathbf{q}}=f_{\mathbf{q}}(\mathbf{q}, \mathbf{Q}, \nabla T, \nabla \mathbf{q}, \nabla \mathbf{Q}, \cdots),
$$

$$
\dot{\mathbf{Q}}=f_{\mathbf{Q}}(\mathbf{q}, \mathbf{Q}, \nabla T, \nabla \mathbf{q}, \nabla \mathbf{Q}, \cdots) \text {. }
$$

The time derivatives of $\mathbf{q}$ and $\mathbf{Q}$ appear respectively in $X_{\mathbf{q}}$ and $X_{\mathbf{Q}}$, in such a way that the evolution equations for them may be achieved by expressing $X_{\mathbf{q}}$ and $X_{\mathbf{Q}}$ in terms of $\mathbf{q}$ and $\mathbf{Q}$. Therefore the following nonlinear thermodynamic force-flux relations are proposed:

$$
X_{\mathbf{q}}=\mu_{1} \mathbf{q}+\mu_{12} \mathbf{Q} \cdot \mathbf{q},
$$

with $\mu_{1}, \mu_{2}, \mu_{12}$ and $\mu_{21}$ being phenomenological coefficients. In usual linear formulations of EIT, it is assumed that $\mu_{12}=0, \mu_{21}=0$ and linear heat transport equation is derived. However, Eq. (1) is nonlinear, thus here the nonlinear terms must be considered as well. Since we have a vector $\mathbf{q}$ and a tensor $\mathbf{Q}, \mathbf{q}$ and $\mathbf{Q} \cdot \mathbf{q}$ arise as two independent vectors, whereas $\mathbf{Q}$ and $\mathbf{q q}$ as two independent tensors. Based on the Curie's principle (requiring that in isotropic systems, vectorial effects must be related to vectorial causes, and tensorial effects to tensorial causes [2]), the vectorial force $X_{\mathbf{q}}$ will depend on the vectors $\mathbf{q}$ and $\mathbf{Q} \cdot \mathbf{q}$, while the tensorial force $X_{\mathbf{Q}}$ will depend on the tensors $\mathbf{Q}$ and $\mathbf{q q}$. Notice that $\mathbf{Q}$ could be split into two independent parts as $\mathbf{Q}=\mathbf{Q}_{0}+Q \mathbf{I}$ with $Q$ the trace of $\mathbf{Q}$ and $\mathbf{Q}_{0}$ the deviatoric part. In this case, the vectors appearing in the constitutive equations will be $\mathbf{q}$, $Q \mathbf{q}, \mathbf{Q}_{0} \cdot \mathbf{q}$ and the tensors $Q \mathbf{I}, \mathbf{q q}, \mathbf{Q}_{0}$. For the sake of simplicity, however, we will stick to the simplest approach, which is sufficient for conceptual clarification and physical interpretation.

Therefore by substituting Eq. (12) and Eq. (13) into Eq. (7), the entropy production and then the entropy inequality (second law) becomes:

$$
\sigma^{s}=\mu_{1} \mathbf{q} \cdot \mathbf{q}+\mu_{12} \mathbf{Q}: \mathbf{q q}+\mu_{2} \mathbf{Q}: \mathbf{Q}+\mu_{21} \mathbf{Q}: \mathbf{q q} \geq 0 .
$$

Thus we have the following restrictions on the phenomenological coefficients to ensure a positive-definite entropy production for arbitrary values of $\mathbf{q}$ and $\mathbf{Q}$ :

$$
\mu_{1} \geq 0, \mu_{2} \geq 0, \mu_{12}=-\mu_{21}
$$




\section{Generalized irreversible thermodynamics}

Combined with Eq. (8) and Eq. (9), Eq. (12) and Eq. (13) turn into respectively:

$$
\begin{gathered}
-\alpha_{1} \dot{\mathbf{q}}+\nabla\left(\theta^{-1}\right)+\beta \nabla \cdot \mathbf{Q}=\mu_{1} \mathbf{q}+\mu_{12} \mathbf{Q} \cdot \mathbf{q}, \\
-\alpha_{2} \dot{\mathbf{Q}}+\beta \nabla \mathbf{q}=\mu_{2} \mathbf{Q}+\mu_{21} \mathbf{q q} .
\end{gathered}
$$

These are exactly the evolution equations for $\mathbf{q}$ and $\mathbf{Q}$, since they yield the time derivatives of $\mathbf{q}$ and $\mathbf{Q}$ as Eq. (10) and Eq. (11). Up to here, the development is general (up to second order in $\mathbf{q}$ and $\mathbf{Q}$ ). In the next section, we will specify the form of $\mathbf{Q}$ to obtain the explicit heat transport equation.

\section{Flux of the heat flux}

To relate the current thermodynamic formalism to heat transport equation Eq. (1), it is necessary to express $\mathbf{Q}$ in terms of $\mathbf{q}$. This will be possible when the relaxation time of $\mathbf{Q}$ is negligible in comparison with the relaxation time of the heat flux, i.e. $\alpha_{2} \ll \alpha_{1}$ in which case $\mathbf{Q}$ is no longer an independent variable on its own, but a variable dependent on $\mathbf{q}$ through $\nabla \mathbf{q}$ and qq based on Eq. (17). Though, for $\mu_{12}=-\mu_{21}=0$ and non-negligible $\alpha_{2}$, Eq. (16) reduces to the generalized Guyer-Krumhansl model [21] when combined with Eq. (17). For $\mu_{12}=-\mu_{21}=0$ and negligible $\alpha_{2}$ as well, Eq. (16) will reduce to the Guyer-Krumhansl model (phonon hydrodynamics model) $[28,29]$. For $\mu_{12}=-\mu_{21} \neq 0$ and negligible $\alpha_{2}$ in present work, the flux of heat flux $\mathbf{Q}$ could be got from Eq. (17):

$$
\mathbf{Q} \simeq \frac{\beta}{\mu_{2}} \nabla \mathbf{q}-\frac{\mu_{21}}{\mu_{2}} \mathbf{q q},
$$

and we obtain by substituting Eq. (18) into Eq. (16):

$\frac{\alpha_{1}}{\mu_{1}} \dot{\mathbf{q}}+\mathbf{q}=-\frac{1}{\mu_{1} \theta^{2}} \nabla \theta-\frac{\beta \mu_{21}}{\mu_{1} \mu_{2}}\left[\mathbf{q} \nabla \cdot \mathbf{q}+\mathbf{q} \cdot \nabla \mathbf{q}-\frac{1}{2} \nabla \mathbf{q}^{2}\right]+\frac{\beta^{2}}{\mu_{1} \mu_{2}} \nabla^{2} \mathbf{q}-\frac{\mu_{21}^{2}}{\mu_{1} \mu_{2}} \mathbf{q}^{2} \mathbf{q}$.

It is seen that Eq. (19) is similar, but not exactly equal to Eq. (1). In particular, it is seen after a careful comparison of Eq. (19) and Eq. (1) that the following equivalences between the coefficients are established:

$$
\tau=\frac{\alpha_{1}}{\mu_{1}} ; \lambda=\frac{1}{\mu_{1} \theta^{2}} ; m_{1}=m_{2}=-2 m_{3}=-\frac{\beta \mu_{21}}{\mu_{1} \mu_{2}} ; m_{4}=\frac{\beta^{2}}{\mu_{1} \mu_{2}} .
$$

Therefore the expression of entropy flux Eq. (6), combined with Eq. (18) and Eq. (20), is specified as:

$$
\mathbf{J}^{s}=\frac{\mathbf{q}}{\theta}+\frac{m_{4}}{\lambda \theta^{2}} \nabla \mathbf{q} \cdot \mathbf{q}+\frac{m_{1}}{\lambda \theta^{2}} \mathbf{q}^{2} \mathbf{q}
$$


Similar generalized expressions of the entropy flux have been proposed in Ref. $[36,38]$, but through a different procedure analogous to rational extended thermodynamics, and for a heat transport equation less general than Eq. (1). The generalized entropy density could be obtained by integrating Eq. (2) and combining Eq. (20):

$$
s(u, \mathbf{q}, \mathbf{Q})=s_{\mathrm{eq}}(u)-\frac{\tau}{2 \rho \lambda \theta^{2}} \mathbf{q}^{2}-\frac{\alpha_{2}}{2 \rho} \mathbf{Q}: \mathbf{Q},
$$

with $s_{\text {eq }}(u)$ the local-equilibrium specific entropy.

Furthermore, the terms in $m_{5}, m_{6}$ and $m_{7}$ in Eq. (1) are still lacking in Eq. (19). Nevertheless, the term in $m_{5}$ could be easily obtained by splitting the flux of heat flux $\mathbf{Q}$ into a deviatoric part and trace part $\mathbf{Q}=\mathbf{Q}_{0}+Q \mathbf{I}$ and taking $u, \mathbf{q}, \mathbf{Q}_{0}$ and $Q$ as state variables [21,33].

\section{Nonlinear thermal conductivity}

The terms in $m_{6}$ and $m_{7}$ in Eq. (1) could also be obtained when we consider in Eq. (12) a more general phenomenological coefficient $\mu_{1}$ as:

$$
\mu_{1}=\mu_{10}\left(\mathbf{I}+a \mathbf{q q}+b \mathbf{q}^{2} \mathbf{I}\right),
$$

with I denoting the unit tensor. Based on Eq. (20), we have $\mu_{1}=1 / \lambda \theta^{2}$ thus Eq. (23) is rewritten in terms of a nonlinear thermal conductivity dependent on $\mathbf{q}$ as:

$$
\lambda=\frac{\lambda_{0} T^{2}}{\theta^{2}}\left(\mathbf{I}+a \mathbf{q q}+b \mathbf{q}^{2} \mathbf{I}\right)^{-1}
$$

with $\mu_{10}=1 / \lambda_{0} T^{2}$. For relatively small values of heat flux, Eq. (24) could be expanded as a form of heat flux-dependent thermal conductivity tensor:

$$
\lambda \approx \lambda_{0}\left(\mathbf{I}-a \mathbf{q q}-b \mathbf{q}^{2} \mathbf{I}\right)
$$

In this way, the terms in $m_{6}$ and $m_{7}$ in Eq. (1) are obtained by substituting Eq. (25) into the extended Fourier law $\mathbf{q}=-\lambda \cdot \nabla \theta$ :

$$
m_{6}=\lambda_{0} a, m_{7}=\lambda_{0} b .
$$

Finally, there is still a nonlinear cubic term in q in Eq. (19), which is lacking in Eq. (1). This is a slight gap of the current thermodynamic framework for the generalized heat transport equation. 


\section{Generalized irreversible thermodynamics}

\section{Conclusions}

The generalized heat transport equation Eq. (1) can be put in a thermodynamic formalism analogous to that of extended irreversible thermodynamics, though a slight gap still exists related to one nonlinear term. A formal approach has been taken here, in order to emphasize the mathematical connections between the several coefficients in the generalized equation Eq. (1) and the coefficients appearing in the expressions of entropy Eq. (2), the entropy flux Eq. (21) and the nonlinear thermal conductivity tensor Eq. (24). The thermodynamic force-flux relations Eq. (12) and Eq. (13) differ from the usual linear ones in classical irreversible thermodynamics and extended irreversible thermodynamics, where the thermodynamic force $\left(X_{\mathbf{q}}\right)$ conjugate to thermodynamic flux $\mathbf{q}$ is not related to thermodynamic flux Q. In the nonlinear domain, however, the vector $X_{\mathbf{q}}$ may be related to both the vectors $\mathbf{q}$ and $\mathbf{Q} \cdot \mathbf{q}$ being still consistent with Curie's principle. Thus here we could consider Eq. (12) and Eq. (13) as extended thermodynamic force-flux relations far from equilibrium states. Nevertheless, up to now the physical meanings of the nonlinear force-flux relations are still ambiguous, which need further investigations in future work. Finally, it should point out that higher-order fluxes could be introduced into the formalism, in such a way that a term $\nabla \cdot \mathbf{Q}^{(3)}$ could appear as an additional contribution to Eq. (17), $\mathbf{Q}^{(3)}$ being the flux of $\mathbf{Q}$, with its own evolution equation. This would result in a hierarchy of evolution equations for higher-order fluxes $\mathbf{Q}^{(n)}$. Linear hierarchies have been already explored in [21], but nonlinear hierarchy will be extremely complicated and still open to exploration.

\section{Acknowledgements}

The authors appreciate the helpful discussions with Professor D. Jou both during and after the visit in Universitat Autònoma de Barcelona, Spain. This work is financially supported by the NSF grant of China (No. 51176089), the Key Basic Scientific Research Program (2013CB228301) and the Specialized Research Fund for the Doctoral Program of Higher Education of China (No. 20130002110077). 


\section{Y. Guo, M. Wang}

\section{REFERENCES}

1. I. Müller, A history of thermodynamics the doctrine of energy and entropy. Heidelberg: Springer, 2007.

2. S. R. De Groot and P. Mazur, Non-equilibrium thermodynamics. New York: Dover Publications, 1962.

3. L. Onsager, Reciprocal relations in irreversible thermodynamics i, Physical Review, vol. 37, pp. 405-426, 1931.

4. L. Onsager, Reciprocal relations in irreversible thermodynamics ii, Physical Review, vol. 38, pp. 2265-2279, 1931.

5. C. Eckart, The thermodynamics of irreversible processes i: The simple fluid, Physical Review, vol. 58, pp. 267-269, 1940.

6. C. Eckart, The thermodynamics of irreversible processes ii: Fluid mixtures, Physical Review, vol. 58, pp. 269-275, 1940.

7. J. Meixner, Zur theorie der irreversiblen prozesse, Annalen der Physik, vol. 5, pp. 244-270, 1943.

8. I. Prigogine, Etude thermodynamique des processus irréversibles. Paris: Desoer, Liege, 1947.

9. T. Qiu and C. Tien, Short-pulse laser heating on metals, International Journal of Heat and Mass Transfer, vol. 35, no. 3, pp. 719-726, 1992.

10. A. Majumdar, Microscale heat conduction in dielectric thin films, Journal of Heat Transfer, vol. 115, pp. 7-16, 1993.

11. D. Y. Tzou, Macro- to microscale heat transfer: the lagging behavior. United Kingdom: John Wiley \& Sons, Ltd., 2015.

12. C. Tien, A. Majumdar, and F. M. Gerner, Microscale energy transport. Washington: Taylor \& Francis, 1998.

13. G. Chen, Nanoscale energy transport and conversion: a parallel treatment of electrons, molecules, phonons, and photons. New York: Oxford University Press, 2005.

14. Z. M. Zhang, Nano/microscale heat transfer. New York: McGraw-Hill, 2007.

15. C. Truesdell, Rational thermodynamics. New York: McGraw Hill, 1969.

16. I. Müller, Zum paradoxon der wärmeleitungstheorie, Zeitschrift der Physik, vol. 198, pp. 329-344, 1967.

17. I.-S. Liu and I. Müller, Extended thermodynamics of classical and degenerate gases. archive for rational mechanics and analysis, Archive for Rational Mechanics and Analysis, vol. 83, pp. 285-332, 1983.

18. I. Müller and T. Ruggeri, Extended thermodynamcis. New York: Springer, 1993.

19. I. Müller and T. Ruggeri, Rational extended thermodynamics. New 


\section{Generalized irreversible thermodynamics}

York: Springer, 1998.

20. D. Jou, J. Casas-Vázquez, and G. Lebon, Extended irreversible thermodynamics, Report on Progress in Physics, vol. 51, pp. 1105-1179, 1988.

21. D. Jou, J. Casas-Vázquez, and G. Lebon, Extended irreversible thermodynamics. Heidelberg: Springer, 2010.

22. P. Ván and T. Fülöp, Universality in heat conduction theory: weakly nonlocal thermodynamics, Annalen der Physik, vol. 524, no. 8, pp. 470478, 2012.

23. P. Ván, B. Czél, T. Fülöp, G. Gróf, A. Gyenis, and J. Verhás, Experimental aspects of heat condution beyond fourier, July 1-5 2013.

24. M. Grmela and H. Öttinger, Dynamics and thermodynamics of complex fluids. i. development of a general formalism, Physical Review E, vol. 56, no. 6, pp. 6620-6632, 1997.

25. H. Öttinger and M. Grmela, Dynamics and thermodynamics of complex fluids. ii. illustrations of a general formalism, Physical Review E, vol. 56, no. 6, pp. 6633-6655, 1997.

26. H. Öttinger, Beyond the equilibrium thermodynamics. Hoboken, New Jersey: John Wiley \& Sons, Inc., 2005.

27. Y. Guo, D. Jou, and M. Wang, Macroscopic heat transport equation and heat waves in nonequilibrium states, To be published.

28. R. Guyer and J. Krumhansl, Solution of the linearized phonon boltzmann equation, Physical Review, vol. 148, no. 2, pp. 766-788, 1966.

29. R. Guyer and J. Krumhansl, Thermal conductivity, second sound, and phonon hydrodynamic phenomena in nonmetallic crystals, Physical Review, vol. 148, no. 2, pp. 778-788, 1966.

30. D. Y. Tzou, The generalized lagging response in small-scale and highrate heating, International Journal of Heat and Mass Transfer, vol. 38, no. 17, pp. 3231-3240, 1995.

31. B.-Y. Cao and Z.-Y. Guo, Equation of motion of a phonon gas and non-fourier heat conduction, Journal of Applied Physics, vol. 102, no. 5, p. 053503, 2007.

32. M. Wang, N. Yang, and Z.-Y. Guo, Non-fourier heat conductions in nanomaterials, Journal of Applied Physics, vol. 110, no. 6, p. 064310, 2011.

33. D. Jou and J. Casas-Vázquez, Nonequilibrium absolute temperature, thermal waves and phonon hydrodynamics, Physica A, vol. 163, no. 1, pp. 47-58, 1990.

34. W. Dreyer and H. Struchtrup, Heat pulse experiments revisited, Continuum Mechanics and Thermodynamics, vol. 5, no. 1, pp. 3-50, 1993. 


\section{Y. Guo, M. Wang}

35. G. Lebon and P. Dauby, Heat transport in dielectric crystals at low temperature: A variational formulation based on extended irreversible thermodynamics, Physical Review A, vol. 42, no. 8, pp. 4710-4715, 1990.

36. G. Lebon, D. Jou, J. Casas-Vázquez, and W. Muschik, Weakly nonlocal and nonlinear heat transport in rigid solids, Journal of Non-Equilibrium Thermodynamics, vol. 23, no. 2, pp. 176-191, 1998.

37. D. Jou, G. Lebon, M. S. Mongiovi, and R. A. Peruzza, Entropy flux in non-equilibrium thermodynamics, Physica A, vol. 338, no. 3-4, pp. 445457, 2004.

38. V. Cimmelli, A. Sellitto, and D. Jou, Nonlinear evolution and stability of the heat flow in nanosystems: Beyond linear phonon hydrodynamics, Physical Review B, vol. 82, no. 18, p. 184302, 2010.

39. D. Jou and L. Restuccia, Mesoscopic transport equations and contemporary thermodynamics: an introduction, Contemporary Physics, vol. 52, no. 5, pp. 465-474, 2011.

40. A. Sellitto, V. A. Cimmelli, and D. Jou, Entropy flux and anomalous axial heat transport at the nanoscale, Physical Review B, vol. 87, no. 5, p. 054302, 2013.

41. G. Lebon, Heat conduction at micro and nanoscales: A review through the prism of extended irreversible thermodynamics, Journal of NonEquilibrium Thermodynamics, vol. 39, no. 1, pp. 35-59, 2014.

42. V. A. Cimmelli, D. Jou, T. Ruggeri, and P. Ván, Entropy principle and recent results in non-equilibrium theories, Entropy, vol. 16, no. 3, pp. 1756-1807, 2014. 